\title{
On Indirectness in English and Chinese Advertising Language from the Perspective of Semiotics
}

\author{
${ }^{1}$ Yanni Li, ${ }^{1}$ Jingyu Yang, ${ }^{2}$ Jiayin Li, ${ }^{1}$ Yiqun Liu, ${ }^{1}$ Peiyu Li \\ ${ }^{1}$ School of Foreign Languages, Changchun Institute of Technology, Jilin 130012, China; \\ ${ }^{2}$ Jilin Animation Institute, School of Communication, Jilin 130012, China \\ aliyanni2007@163.com
}

Keywords: advertising language; semiotics; indirectness; presupposition; pun

\begin{abstract}
In this economic society, advertising has overwhelmingly penetrated every corner of our life. As a way of transmitting information, advertising's role cannot be neglected. As for advertising, language is the most inevitable part, which is worthy serious research. In the field of linguistics, many scholars have achieved outstanding achievement on advertising language from various perspectives. However, it's still scare that analyzing advertising language from the semiotics perspective, especially focusing on the indirectness of advertising language, which is a common phenomenon in advertising, since indirect expressions are euphemistic, leaving some room for people to imagine and consider, and showing respect on them, it is easier to achieve better effects. Semiotics as the study of signs concerns the exchange of messages and the systems of signs that underlie them. The discipline of semiotics may be used to predict what the advertisement may mean to the audience, to determine if the words or visual material will be understood by the public in the way that the advertiser wish. The theory and concepts of semiotics may also be used to analyze the structure of advertising language so as to obtain a better understanding how it works.
\end{abstract}

\section{Introduction}

Advertisement is the mirror of society. It has influenced every aspect of society: arts, culture, sport, fashion, politics and even religion. The reason for this phenomenon is that nothing can survive in a competitive market without advertising itself. Further more, advertisements yield great economic and cultural power in dictating values, lifestyles and consumer trends. Advertisements can appear in any form: TV, radio, film, print, Internet, and outdoor formats (billboards, bus and taxi, dust bins, posters, buildings, etc.) People are overwhelmed by ads in their daily life.

What's more, much attention is attached to the research of advertising language because proper advertising language can help establish good image for corporations or manufactures by various kinds of languages signs. In spite of those great achievements on adverting language, there is little about indirect expression in it. Due to the consumers' psychology and certain social factors, advertisers are getting more and more interested in indirect way to express their intentions. From the semiotic perspective, signs are the nature of language. Each sign involves two aspects, signifier and signified, and the relationship between them is relatively arbitrary. In advertising language, signifier refers to literal form, and signified refers to the connotation of the language. This approach enables people to find the necessity and reasons of indirectness, as well as some clues to interpret indirect expressions in advertising language.

\section{The semiotic features of indirectness in advertising language}

From Saussure's view, we have learned that the relationship between signifier and the signified is relatively arbitrary, not absolutely arbitrary. As a means of communication, advertisement can be considered as a sign operation process. Semiotics, as the study of signs, mainly concerns the exchange of messages and the systems of signs that underlie them[1]. Therefore, the features of semiotics may be found in indirectness in advertising language as well. 


\section{1) The "relatively arbitrary" relationship between signifier and signified}

As one of the best semiotic analysis of advertising, Judith Willianson's Decoding Advertisements did a great contribution to the advertisement studies. She uses the ad for the French perfume Chanel to illustrate her arguments: the face of a woman (a French fashion model) is shown with a picture of the product (a bottle of Chanel No.5) in the corner of the image. We may wonder what the meaning of this ad is. It might be: Chanel No.5 is sophisticated and elegant, and by wearing it we could add something "Frenchness", especially, glamour and flawless beauty. When we analyze this ad from the semiotic angle, we have the signifier: French "Chanel No.5" and a fashion model, and the signified French chic, glamour, beauty and sophistication. Assuming that this is the ad meant to us, and then we may ask: How do we arrive at that conclusion?

The combination and relationship of the signifier and signified seem arbitrary, the advertisers could choose other ways to advertise their products. For example, they can choose a man or a famous foreign star instead of that French lady model. However, after comparison, people would still consider that the original one is perfect, because considering the signified is chic, beauty, elegant and supplicated, and the cultural background of the market, the signifier is fixed to a French beauty. We can conclude that the relationship between the signifier and the signified in indirectness is relatively arbitrary, which is determined by the context and cultural background.

\section{2) Two levels of meaning}

Further more, although nothing in this kind of ad is explicitly stated, we can find there are two levels of meaning conveyed, that is, literal meaning and non-literal meaning. In semiotic perspective, they are denotative meaning and connotative meaning. The meaning of such an ad does not just float on the surface, waiting to be internalized by the people, but is built up out of ways that different signs are organized and related to each other[2], both within the ad and through external references to wider belief systems. More specifically, people have to do some work to get the meaning of the ad, because the meaning is not lying on the page, we have to make efforts to grasp it.

Because of the above semiotic features of indirectness in advertising language, advertisers have to pay attention to the following three factors when designing advertisements: First, the meaning of one sign is transferred to another. There are many ways this transfer can take place, such as between persons and objects, between social situations and objects, between objects and objects, and between feelings and objects, etc. Secondly, the process of transfer is not completed within the ad, that is to say, people must make the connection between them by themselves. Therefore, advertisers have to take consumers' cognition ability into consideration. Thirdly, in order to make the transfer go smoothly, the signifier must already have a meaning to be transferred, which is acceptable and familiar to the audience. In other words, the advertisers have to study the culture background of the target audience, so that the ads could be understood in the way they wish and finally realize their promotion purpose.

Based on the above knowledge, the author would like to analyze several actual English and Chinese advertisements language, mainly focusing on indirectness, from the perspective of semiotics, with the hope of providing something different in advertising studies.

\section{Case-analysis on indirectness in advertising language from the perspective of semiotics}

From the previous parts, we can learn that advertisers widely employ indirect ways to express their intentions. From one hand, this phenomenon is caused by consumers' psychology and culture background; from the other hand, advertisers take advantage of this means in order to perusing aesthetic pleasure and avoid negative social reputations. Under such circumstance, it becomes significant that to analyze the fundamental form and meaning of indirectness in advertising language. Based on the semiotic features of indirectness in advertising language, we can analyze this phenomenon in a new angle. As we know, from the angle of semiotics, the signifier refers to the form of advertising language. After comparison and collection, it comes out that the signifier of such ads can be divided into two categories, that is, presupposition and pun. In this part, the author will provide simple explanation of these two groups. Moreover, the author has picked up several actual ads from our daily life to illustrate her explanations. 


\section{1) Presuppositions}

In the modern society, under such fierce competition, advertisers have to adopt some novel ideas when creating ads, since they have to bear the advertising expenditures, and pay for the advertising space and choice of advertising words. Therefore, the advertisers need advertising language to be concise. Presuppositions make language concise, because they are dependent on people's knowledge and commonsense: those things don't need to be spoken out. For example: "Pete's sister is pretty" presupposes that "Pete has a sister". The advertisers can make some parts of information as presupposed information, so that making advertising language concise.

A presupposition can be "something the speaker assumes to be the case prior to making up an utterance”. From that, we can learn that presupposition enable people to make implicit assumptions of the real world[3]. Here we just focus on the structure of this phenomenon and how it conveys its connotative meaning through the combination of signifier and the signified. Let's discuss some examples from daily life.

Signifier: The nutritious oral liquid does not contain any sex hormone. (Nutritious oral liquid)

Signified: Sex hormone is harmful to the human body;

Other brands of the same kind contain sex hormone. The advertisers imply indirectly that sex hormone is not good, and the nutritious oral liquid which doesn't contain sex hormone is good for people's health. Although they don't say what they mean directly, people can infer the signified from their basic knowledge. Through employing presupposition, advertiser expresses his intention euphemistically, so as to avoid negative social responsibilities.

Signified: There is a noise from the electric clock when Rolls Royce travels at sixty miles an hour.

This ad is very special, because it explodes the shortcoming of product which rarely to find in ads. Therefore, it is different from those the excellent function, high-quality ads, which are common to all of us. However, this ad can attract people's attention easily. And people will find the noise is reasonable, which doesn't affect the function of the car at all. On the contrary, it reflects the superior quality of the product. Moreover, the ad is able to leave a deep impression in consumers' mind.

From the above examples, it is easy to learn that through the concept (the signified) in ads remains the same, the form (the signifier) varies. When employing presupposition, the signified can be worked out from people's knowledge and cultural background. However, presupposition works only if the signifier is meaningful for local consumers. Otherwise, improper usage will sure cause great loses for advertisers.

\section{2) Puns}

According to Longman Dictionary of Contemporary English, "A pun is an assuming use of a word or phrase that two meanings, or of words with the same sound but different meanings." For example, seven days without water make one weak. Some scholars believe that a pun is a play on word, based on the identity or similarity between the sounds of words with different meanings. The main use of pun is to make jokes, leaving a deep impression on people. Due to its unique function, advertisers like to employ puns in advertising language.

As we have mentioned before, according to semiotics, there are two levels of meaning a sign conveys, namely, connotative meaning and denotative meaning. Denotative meaning refers to the hidden meaning. Similarly, a pun usually has two levels of meaning, while the focus of the users is put on the hidden meaning, not the literal meaning. And the relationship between them is relatively arbitrary. When people read the signifier, they will naturally have an association with the signified. What's more, with a pun in an advertisement, it becomes easier for people to remember the advertised product, and directly stimulate their desire for purchase. It brings people a kind of aesthetic pleasure in reading such a humorous ad. Therefore, some examples are as following:

(1) 只要是美的，人人都喜欢（Everyone loves it if it is Midea.) (Ad for Midea Air conditioner, Canton Evening Newspaper, 1996)

In this advertisement, the Chinese characters “美的” is the brand name for commercial products. This is nothing striking or different; however, what is special is that “美的” is a pun which can also be read as "something beautiful”. It is well-known that a pun is often implicit, which enables people 
have some kinds of association. Midea Company makes full use of such a pronunciation, so that the advertising language has both its literal meaning and conveyed meaning.

(2) We have the edge you need to meet any financial challenge. -----The Principle Edge

It is easy for us to find that "edge" is the key word in this ad. We all know it has the meaning of "the edge of knife'. After reading this ad, people will realize that there is no connection between the ad and a knife. This ad is for the Principle Financial Group, and 'The Principle Edge' is a service name adopted by the Principle Financial Group. Here is a pun used. The title "we have the edge you need to meet any financial challenge." means that we have the superiority you need to meet financial challenge. 'Have the edge' means 'has the superiority' as well. The word 'edge' also refers to the name of the financial service offered by this company.

From the above examples, it is clear that a pun needs longer time to comprehend. Therefore, it can sustain the audiences' attention over a period of time, and when it's comprehended finally, it is difficult to forget. In short, the use of puns ensures that the message is attractive to people. For advertisers, it is crucial to attract the attention of audience. "The audience gains extra contextual effects based on the pleasure and satisfaction of having solved the pun. These effects may affect the audience's attitude towards the ad, and ultimately, the product advertised"[4].To conclude, we can easily find the semiotic features of indirectness in advertising language. No matter which way is employed to express indirectness in ads, presupposition or pun, we can analyze it from the semiotic perspective. It is found that there are two levels of meaning in indirectness, and the relationship between the signifier and the signified is relatively arbitrary. People's daily knowledge and cultural background have a great impact on the interpretation process.

\section{Summary}

From the above, we can see that with the development of the modern society, advertisements are getting more and more important, and it has become an essential part of our daily life. As we know, ideally, ads should meet the following model. That is: it has to gain the attention of the audience; it has to arouse some level of interest in the company's product or service; it has to nourish desire by giving information about the important benefits of the products or service; it has to promote the action of purchases. Through research and analysis, the author concludes that whether the products advertised in ads attract people's attention or not, whether they win people's impression, or not, depends on the expression of advertising language and understanding of consumers. When making advertisements, advertisers should take the following factors into consideration: Firstly, consumers' cognitive abilities, therefore, advertising language should be simple and easy to understand; secondly, the cultural background of the target market, it is the crucial factor for international ads. The advertisers should make a thorough research on local culture, so as to avoid culture conflicts.

\section{Acknowledgements}

The authors would like to give their thanks to The Education Department of Jilin Province (NO. 2015-201) and National Program of Business Startups for University Students (2015-31; 2015-32) for their financial and technical support.

\section{References}

[1]. David. W. Carrol. Psychology of Language (Third edition). Beijing: Foreign Language Teaching and Research Press, 2001, p33-35.

[2]. Ilze Bezuidenhout. A Discursive-Semiotic Approach to Translating Cultural Aspects Persuasive Advertisements. Beijing: Foreign Language Teaching and Research Press, 1998, p50-52.

[3]. Meng Lin, Zhan Jinghui. Punning and the Translation of Puns in English Advertisements. Zhejing: Journal of South-Central University for Nationalities. (Humanities \& Social Science), 2001, p78-82. 
[4]. Gu Songqing. Social semiotics and analysis of advertising language. Journal of Anhui Science and Technolgy, 2005, 2, p10-15. 